\title{
HUtrevista
}

\section{Comparação de diferentes planejamentos em cirurgia ortognática para tratamento de assimetrias faciais: relato de casos}

- Daniel Amaral Alves Marlière ${ }^{1}$, Hugo Leite Rodrigues Neto ${ }^{2}$ Alan Robert Moreira Schmitt ${ }^{3}$, Luciana Asprino ${ }^{1}$, Rodrigo Alvitos Pereira ${ }^{3}$

\footnotetext{
${ }^{1}$ Área de Cirurgia e Traumatologia Bucomaxilofacial, Faculdade de Odontologia de Piracicaba, Universidade Estadual de Campinas, FOP - UNICAMP, Piracicaba, SP.

2 Faculdade de Odontologia, Hospital e Maternidade Therezinha de Jesus, Faculdade de Ciências Médicas e da Saúde de Juiz de Fora, FCMS/JF - SUPREMA, Juiz de Fora, MG.

${ }^{3}$ Serviço de Cirurgia e Traumatologia Bucomaxilofacial, Hospital Universitário Pedro Ernesto, Universidade do Estado do Rio de Janeiro, HUPE - UERJ, Rio de Janeiro, RJ.
}

\section{Daniel Marlière} Rua Professora Violeta Santos, 31/803, bloco 01 Democrata CEP: $36035-210$ Juiz de Fora - MG ] ctbmf.marliere@gmail.com

\section{RESUMO}

Introdução: a cirurgia ortognática envolve a correção de desarmonias funcionais e estéticas. A hiperplasia condilar ( $\mathrm{HC}$ ) unilateral resulta em crescimento mandibular acentuado, provocando assimetria facial. Para obter previsibilidade e sucesso nos resultados, é imprescindível planejamento preciso e adequado. Objetivo: descrever e comparar diferentes métodos de planejamento e seus resultados no pós-operatório no tratamento de pacientes com assimetria facial. Relato de caso: foram avaliados 03 casos orto-cirúrgicos ( $A, B$ e $C$ ) de pacientes adultos jovens, com média de idade de 22 anos, portadores de má oclusão esquelética de Classe III, com assimetria facial resultante de $\mathrm{HC}$ inativa. Foi realizada análise facial e empregou-se dois métodos de planejamento em cirurgia ortognática (convencional e virtual). O caso A foi planejado por meio de imagens bidimensionais (2D) e sequência cirúrgica iniciada pela maxila. O caso B foi planejado por imagens 2D associado ao recurso de simulação em protótipo. Por fim, o caso $\mathrm{C}$ foi planejado exclusivamente com imagens tridimensionais (3D) e simulação virtual, sendo que os casos B e C utilizaram sequência cirúrgica iniciada pela mandíbula. Foi utilizado o software Dolphin Imaging $®$ em todos os planejamentos e os mesmos foram transferidos para os procedimentos cirúrgicos com auxílio dos guias em resina acrílica estereolitográfica. Conclusão: os planejamentos demonstraram eficácia em termos de previsibilidade e viabilidade para a correção das assimetrias faciais, sendo todos os resultados considerados satisfatórios por parte dos cirurgiões e dos pacientes. Houve tendência de melhores resultados quando da utilização de prototipagem, das imagens 3D e sequência operatória iniciada pela mandíbula, no planejamento.

Palavras-chave: assimetria facial, cirurgia ortognática, côndilo mandibular

\section{ABSTRACT}

Introduction: orthognathic surgery provides correction of functional and esthetic disharmony. Condylar hyperplasia induces in pronounced mandibular growth, resulting facial asymmetry. Planning is important to get predictable and successful on outcomes. Purpose: to describe and compare methods of planning and post-operative outcomes by reporting three cases associated with facial asymmetries. Case report: a total of 03 young adult patients (cases A, B and C), mean of age 22 years old, Class III skeletal malocclusion, and facial asymmetry after inactive condylar hyperplasia were evaluated. They were undergone facial analysis, two methods of planning in orthognathic surgery (conventional and virtual). Patient A was planned by bidimensional (2D) images and maxilla first sequence. Patient $B$ was planned by 2D images associated with simulating on surface mesh prototyping. And, Patient $C$ was solely planned by three-dimensional (3D) images and virtual surgical planning. Patients $B$ and $C$ used mandible first sequence. Dolphin Imaging software was used on each planning. Each planning was transferred to surgical procedures by assisting of the guides manufactured (acrylic resin and stereolithography). Patients were usually assessed during post-operative and were pleasured with outcomes. Conclusion: planning showed predictable and feasibility for facial asymmetries because outcomes were great. There was a propensity of better outcomes when surface mesh, 3D images and mandible first sequence were used at the treatment planning.

Keywords: facial asymmetry, orthognathic surgery, mandibular condyle 


\section{INTRODUÇÃO}

A simetria facial perfeita é raramente encontrada, devido a fatores biológicos e ambientais inerentes aos processos de desenvolvimento, que podem gerar diferentes graus de assimetria leves e imperceptíveis (THIESEN; GRIBEL; FREITAS, 2015). No entanto, os quadros clínicos classificados como assimetria facial consistem em variação significativa entre as duas metades da face que, quando exacerbada, propicia alterações estéticas e problemas funcionais quanto ao desenvolvimento orofacial, nutricional e psicossocial nos pacientes (SRIVASTAVA et al., 2018).

Segundo Good et al. (2006), a má oclusão de Classe III está frequentemente associada aos casos de assimetria facial. A relação entre tais condições pode ter como fator etiológico a hiperplasia de côndilo (HC) unilateral, que predispõe a piora progressiva da má-oclusão, com a presença de mordida cruzada contralateral, desvio mandibular e mentual para o lado oposto e desnivelamento oclusal (SRIVASTAVA et al., 2018).

Dados epidemiológicos demonstram ocorrência significante de casos de assimetria de face com distribuição igual entre homens e mulheres (RAMIREZYAÑEZ et al., 2011). De acordo com Wolford et al. (2009), o superdesenvolvimento mandibular simétrico ou desviado possui maior ocorrência durante a puberdade, podendo o crescimento ocorrer até os 20 anos. O acompanhamento e o diagnóstico dos casos de assimetria de face podem ser realizados pelos exames clínicos, pela análise da face (fotografias para verificação das proporções faciais), dos modelos de gesso e de exames de imagens (MARLIÈRE et al., 2017; LÓPEZ; CORRAL, 2015).

A cirurgia ortognática é a conduta de escolha para correção de assimetria facial por demonstrar estabilidade em longo prazo e melhora da estética e função mastigatória do paciente (SINGH et al., 2017). A previsibilidade dos resultados cirúrgicos está associada a alguns recursos, tais como de exames clínicos adequados, imagens bidimensionais (2D) e/ou tridimensionais (3D) e ferramentas específicas de planejamento (MARLIĖRE et al., 2018).

No passado, os planejamentos em cirurgia ortognática eram realizados exclusivamente por meio de imagens $2 \mathrm{D}$ e simulação em articuladores semi-ajustáveis (ASA), visando à previsibilidade de posicionamento dos maxilares e auxiliar a transferência do planejamento para o procedimento por meio da confecção de guias cirúrgicos (SUN et al., 2013). Com as inovações tecnológicas, os planejamentos avançaram proporcionalmente aos métodos de imagem, como nas tomografias computadorizadas e com os programas computacionais para segmentação de imagens tomográficas e renderização 3D, confecção de protótipos estereolitográficos e simulações cirúrgicas em ambiente virtual, que se tornaram úteis e eficazes (HASS JR; BECKER;
OLIVEIRA, 2015; RIU et al., 2014).

Portanto, o objetivo deste estudo foi descrever e comparar a eficácia de diferentes métodos de planejamento (convencional e virtual) e os resultados obtidos no tratamento de 03 pacientes assimétricos submetidos à cirurgia ortognática.

\section{RELATO DOS CASOS}

Três pacientes compareceram ao Serviço de Cirurgia Bucomaxilofacial do Hospital Universitário Pedro Ernesto (Universidade do Estado do Rio de Janeiro, Rio de Janeiro) para tratamento de deformidade dentofacial e má-oclusão Classe III, queixando-se da aparência estética facial e restrições funcionais. Todos os pacientes foram submetidos a exame clínico intrabucal, análise facial associado a fotografias faciais, exames tomográficos e confecção de modelos de gesso dos arcos dentários de maxila e mandíbula.

Para a análise facial, cada paciente foi analisado quanto aos contornos de tecido mole facial, de estruturas ósseas e dentárias. A função das articulações temporomandibulares também foi avaliada. Dessa forma, foi compreendido que a etiologia da assimetria facial possivelmente foi por $\mathrm{HC}$ unilateral. Os pacientes foram acompanhados clinicamente por 06 meses para confirmação de inatividade de crescimento assimétrico.

Durante anamnese, os pacientes não relataram nenhuma alteração sistêmica ou fatores de comorbidades, sendo solicitados exames laboratoriais pré-operatórios (hemograma, coagulograma e glicemia em jejum). Os resultados dos exames não indicaram qualquer anormalidade. Os pacientes assinaram o termo de ciência e consentimento informado para realização do procedimento cirúrgico e autorizaram a publicação para fins científicos.

\section{Pré-operatório}

\section{Caso $A$}

Paciente de 21 anos foi submetida à análise facial e exame intrabucal. As avaliações demonstraram assimetria facial com má-oclusão Classe III associada a mordidas cruzadas posterior e anterior, mordida aberta anterior de $2,0 \mathrm{~mm}$, overjet de $-11,0 \mathrm{~mm}$, desvio mandibular para lado esquerdo, linha média dentária superior desviada $1,5 \mathrm{~mm}$ para direita e inferior $7 \mathrm{~mm}$ para esquerda, perfil facial côncavo e exposição dentária inadequada em repouso e ao sorrir (Figura 1). As características faciais estão no quadro 1.

\section{Caso $B$}

Jovem de 22 anos foi submetido à análise facial e exame intrabucal. As avaliações demonstraram assimetria facial com má-oclusão Classe III associada a mordida cruzada posterior e anterior, mordida aberta anterior de $6 \mathrm{~mm}$, trespasse horizontal de $-6 \mathrm{~mm}$, com desnivelamento nos 

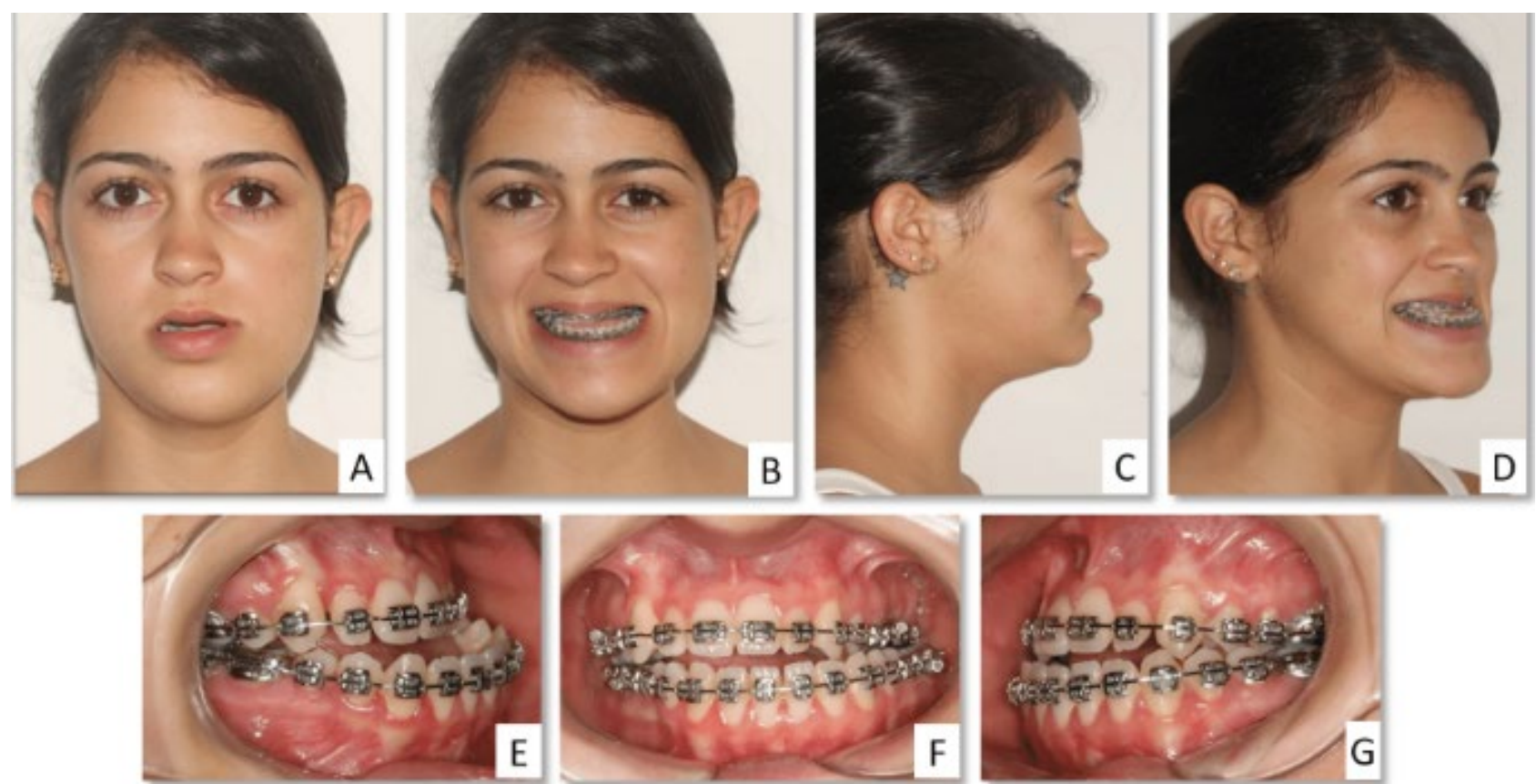

Figura 1: (A-D) Imagens pré-operatórias da face com lábio relaxado e ao sorriso; (E-G) Imagens da oclusão pré-operatórias
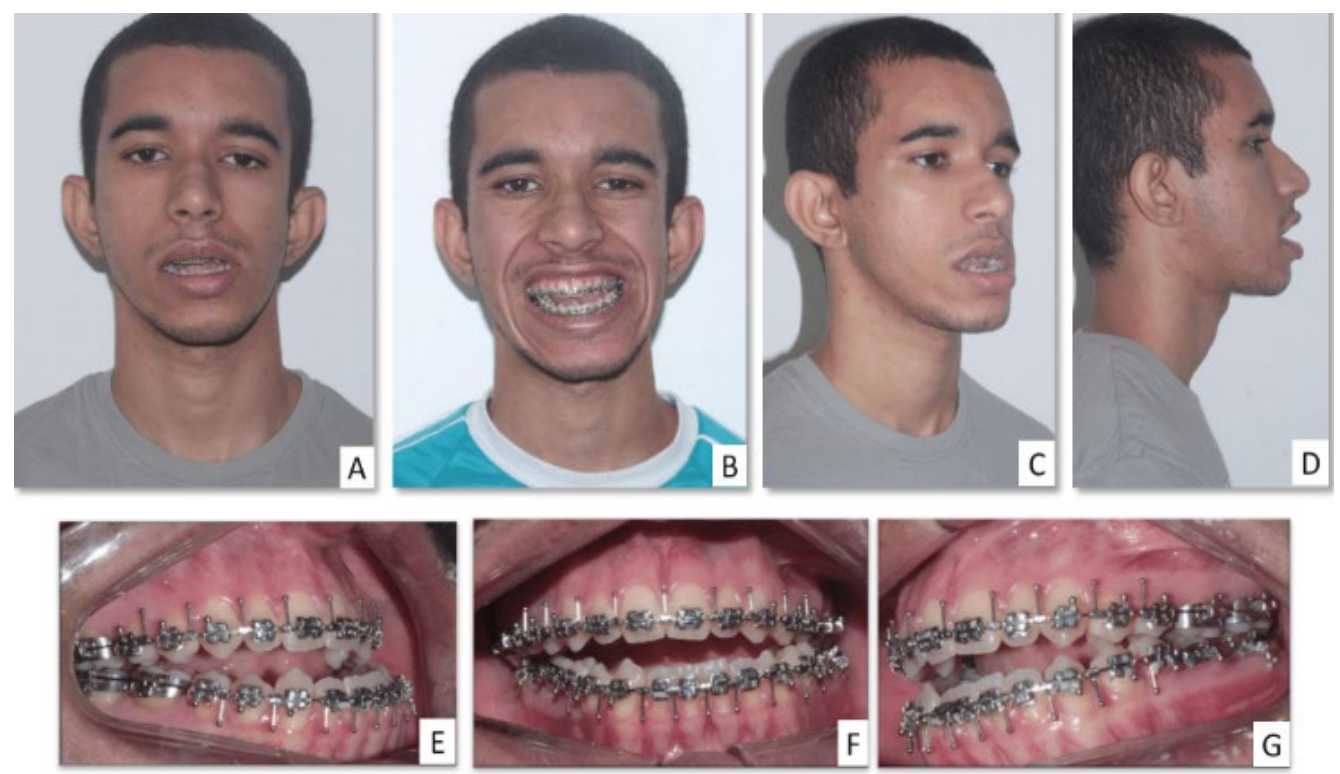

Figura 2: (A-D) Imagens pré-operatórias da face com lábio relaxado e ao sorriso; (E-G) Imagens da oclusão pré-operatórias

caninos superiores e inferiores, desvios de linhas médias dentárias superior de $2 \mathrm{~mm}$ para direita e inferior de 4 $\mathrm{mm}$ para esquerda, maior exposição gengival a direita durante sorriso (Figura 2). As características faciais estão no quadro 1 .

\section{Caso C}

Mulher de 23 anos foi submetida à análise facial e exame intrabucal. A análise facial mostrou aparência de face longa, sem excesso vertical aparente de maxila, com exposição gengival adequada ao sorrir (aproximadamente $2 \mathrm{~mm}$ de margem gengival), juntamente com projeção do mento em relação ao lábio inferior. O exame intrabucal demonstrou má-oclusão classe III, sobremordida normal, overjet de - 9mm, com desvio de linha média superior ( $2 \mathrm{~mm}$ para direita) e inferior (1,5 mm para esquerda) (Figura 3). O quadro 1 descreve as características faciais.

A partir dos exames clínicos e de imagem dos três casos, foi verificado assimetria facial resultante de HC unilateral, que estava inativa após 06 meses de acompanhamento clínico. Os planejamentos para os três casos foram baseados a partir dos dados clínicos, e diferentes métodos de planejamento foram utilizados. 
Planejamento e simulação cirúrgica

Primeiramente, as fotografias faciais pré-operatórias foram registradas em posição natural da cabeça. Para isso, todos os pacientes foram orientados a olhar em direção a um ponto distante ao nível dos olhos e, se necessário, os cirurgiões os reorientavam. Os exames de tomografia computadorizada de feixe cônico (TCFC) foram realizados, nos três pacientes, através do tomográfo i-CAT (Image Sciences International, Hatfield, PA, EUA), com tempo de varredura de 40s, ajuste de $120 \mathrm{kVp}, 5 \mathrm{~mA}$, voxel 0,4 $\mathrm{mm}$ isotrópico e escala de cinza de 14 bits. Os arquivos de imagens no formato DICOM foram importados para o programa computacional Dolphin Imaging ${ }^{\circledR} 11.7$ (Dolphin Imaging and Management Solutions, Chatsworth, CA, EUA).
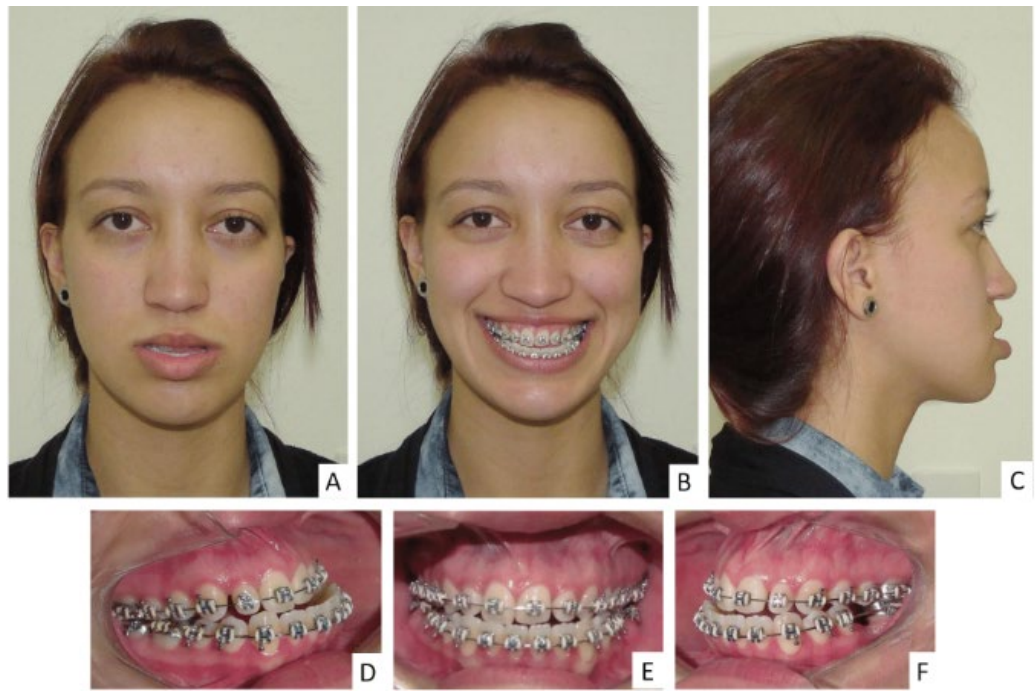

Figura 3: $(A-C)$ Imagens pré-operatórias da face com lábio relaxado e ao sorriso; (D-F) Imagens da oclusão pré-operatórias

Quadro 1: Comparação das características clínicas pacientes dos casos A, B e C

\begin{tabular}{|c|c|c|c|}
\hline \multicolumn{4}{|c|}{ Dados qualitativos da análise facial } \\
\hline Vista frontal & Caso A & Caso B & Caso C \\
\hline & Simétricas & Assimétricas & Simétricas \\
\hline (Simetria pupilar) & Paralelo ao solo & Desnivelado & Paralelo ao solo \\
\hline Nariz & Não desviado & Não desviado & Não desviado \\
\hline Filtro do lábio & Não desviado & Não desviado & Não desviado \\
\hline Linha labial e comissura oral & $\begin{array}{l}\text { Desnivelamento labial e em } \\
\text { comissuras }\end{array}$ & $\begin{array}{l}\text { Desnivelamento labial e em } \\
\text { comissuras }\end{array}$ & $\begin{array}{c}\text { Desnivelamento labial e } \\
\text { em comissuras }\end{array}$ \\
\hline $\begin{array}{l}\text { Linha media do incisivo } \\
\text { superior ao plano mediano } \\
\text { sagital }\end{array}$ & Desviado para a direita & Desviado para a direita & Desviado para a direita \\
\hline Plano oclusal transversal & $\begin{array}{l}\text { Desnivelamento em caninos } \\
\text { superiores (lado direito mais } \\
\text { baixo) }\end{array}$ & $\begin{array}{l}\text { Desnivelamento em caninos } \\
\text { superiores (lado direito mais } \\
\text { baixo) }\end{array}$ & $\begin{array}{c}\text { Desnivelamento em caninos } \\
\text { superiores (lado esquerdo } \\
\text { mais baixo) }\end{array}$ \\
\hline Exposição gengival em sorriso & Sem exposição & Maior exposição à esquerda & Maior exposição à esquerda \\
\hline Linha media do incisivo inferior & Desviada para a esquerda & Desviada para a esquerda & Desviada para a esquerda \\
\hline Lábio inferior e mento & Deslocados para a esquerda & Deslocados para a esquerda & Deslocados para a esquerda \\
\hline $\begin{array}{l}\text { Comprimento do ramo } \\
\text { mandibular }\end{array}$ & Diferença vertical & Diferença vertical & Nenhuma diferença \\
\hline Vista lateral & Caso A & Caso B & Caso C \\
\hline $\begin{array}{l}\text { Suporte do lábio superior } \\
\text { (ângulo nasolabial) }\end{array}$ & $\begin{array}{l}\text { Pouco suporte labial } \\
\text { superior (agudo) }\end{array}$ & $\begin{array}{l}\text { Pouco suporte labial } \\
\text { superior (normal) }\end{array}$ & $\begin{array}{l}\text { Pouco suporte labial } \\
\text { superior (agudo) }\end{array}$ \\
\hline Incisivo superior & Retroposicionado & Retroposicionado & Retroposicionado \\
\hline Preenchimento paranasal & Deficiente & Bom & Deficiente \\
\hline Mandíbula e mento & Prognatismo & Prognatismo & Prognatismo \\
\hline
\end{tabular}


Para os casos A e B, o planejamento convencional foi realizado por meio de exames de imagens bidimensionais (2D), montagem de modelos de gesso em ASA e simulação cirúrgica. Exclusivamente, o planejamento do caso $B$ foi simulado em protótipo confeccionado a partir da TCFC. O caso $\mathrm{C}$ teve seu planejamento realizado de forma tridimensional (3D) e virtual por meio Dolphin Imaging ${ }^{\circledR}$ 11.7 3D (Dolphin Imaging and Management Solutions, Chatworth, CA, USA).

\section{Planejamento convencional}

O planejamento 2D e convencional foi realizado para o caso $A$ através de radiografias panorâmica e anteroposterior de face, e de telerradiografia de perfil produzidas na ferramenta building x-ray no Dolphin Imaging ${ }^{\circledR}$ (Figura 4A - B). Os modelos de gesso dos arcos mandibular e maxilar foram confeccionados e montados em ASA (Bio-Art, São Paulo, Brasil). A partir da análise facial, foram realizados traçados original e preditivo na telerradiografia de perfil, simulando as movimentações cirúrgicas em estruturas ósseas, postura de lábios e contorno de tecido mole em perfil (Figura 4F). Além da correção da assimetria, o planejamento consistiu de rotação horária do complexo maxilomandibular para proporcionar preenchimento em região paranasal, adequada, exposição de incisivos superiores em relação ao lábio em repouso e melhor postura do mento e lábio inferior.

A seguir, um operador simulou as movimentações cirúrgicas por meio dos arcos dentários dos modelos de gesso montados em ASA, que foram conduzidos à nova posição pelas mensurações na plataforma de Erickson, permitindo nova relação maxilomandibular, que foi transferida para a cirurgia ortognática em sequência iniciada pela maxila por meio de guias confeccionados com resina acrílica autopolimerizável (JET, São Paulo, Brasil).

\section{Planejamento convencional e simulação em protótipo}

No caso $B$, o planejamento 2D e convencional foi realizado por meio de radiografias panorâmica, anteroposterior de face e telerradiografia de perfil produzidas na ferramenta building x-ray no Dolphin Imaging $($ (Figura $5 A$ - B). Os modelos de gesso dos arcos mandibular e maxilar foram confeccionados e montados em ASA (Bio-Art, São Paulo, Brasil). A partir da análise facial, foi realizado traçado original e preditivo na telerradiografia de perfil, simulando as movimentações cirúrgicas em estruturas ósseas, postura de lábios e contorno de tecido mole em perfil (Figura 4C). Além da correção da assimetria, o planejamento consistiu de rotação anti-horária do complexo maxilomandibular e mentoplastia para melhorar a postura de lábio inferior em relação ao pogônio.

A seguir, o operador simulou as movimentações cirúrgicas por meio dos arcos dentários dos modelos de gesso montados em ASA (Bio-Art, São Paulo, Brasil) que foram conduzidos a uma nova posição pelas mensurações na plataforma de Erickson, permitindo nova relação maxilomandibular, que foi transferida para a cirurgia ortognática em sequência iniciada pela mandíbula por meio de guias confeccionados com resina acrílica autopolimerizável (JET, São Paulo, Brasil). No protótipo proveniente da reconstrução ou renderização tridimensional (Figura 5D - E), o operador simulou as osteotomias sagital bilateral da mandíbula e Le Fort I de acordo com o que seria realizado no paciente, sendo os segmentos ósseos reposicionados de acordo com a simulação em cirurgia de modelo (Figura 5F).

\section{Planejamento virtual}

Para o caso C, o planejamento cirúrgico foi baseado nas queixas estético-funcional da paciente e na análise facial. A partir da TCFC, as imagens foram reconstruídas tridimensionalmente usando programa Dolphin Imaging $\AA$. De acordo com estes fatores, a cirurgia bimaxilar (maxila e mandíbula) foi planejada através de rotação anti-horária do complexo maxilomandibular com mentoplastia. A osteotomia Le Fort I foi selecionada para avanço e discreta impacção de maxila em região de espinha nasal e incisivos superiores, correção de linha média para esquerda em relação ao filtro do lábio e nivelamento oclusal em caninos e molares. A osteotomia sagital de mandíbula bilateralmente foi escolhida para recuo de mandíbula e mentoplastia para avanço do queixo (Figura 6).

\section{Procedimentos cirúrgicos}

As cirurgias foram definidas de acordo com os planejamentos propostos para os três casos. Os procedimentos cirúrgicos foram realizados sob anestesia geral. No caso A, a cirurgia foi realizada em sequência iniciada pela maxila. $O$ acesso cirúrgico foi realizado por abordagem vestibular e descolamento de mucosa não ceratinizada subperiosteal, estendendo-se de assoalho de fossa nasal até região pterigomaxilar. A osteotomia Le Fort I foi executada utilizando serra reciprocante (Stryker - CORE System) e finalizada com cinzeis e martelo. Após as osteotomias em regiões pterigomaxilares e mobilização da maxila, as paredes da maxila foram regularizadas de acordo com planejamento virtual, utilizando pinça goiva e brocas de desgastes. O guia cirúrgico de resina acrílica autopolimerizável foi inserido em conjunto com acessórios ortodônticos e fio de aço, com objetivo de bloqueio maxilomandibular, estabilizando maxila em posição satisfatória para oclusão ideal. Por fim, a maxila foi posicionada com fixação interna rígida com miniplacas em formato de $L$ bilateralmente nas regiões de zigoticomaxilar e ao redor da abertura piriforme (Sistema 2.0 - Neoface - Neoortho Produtos Ortopédicos). 


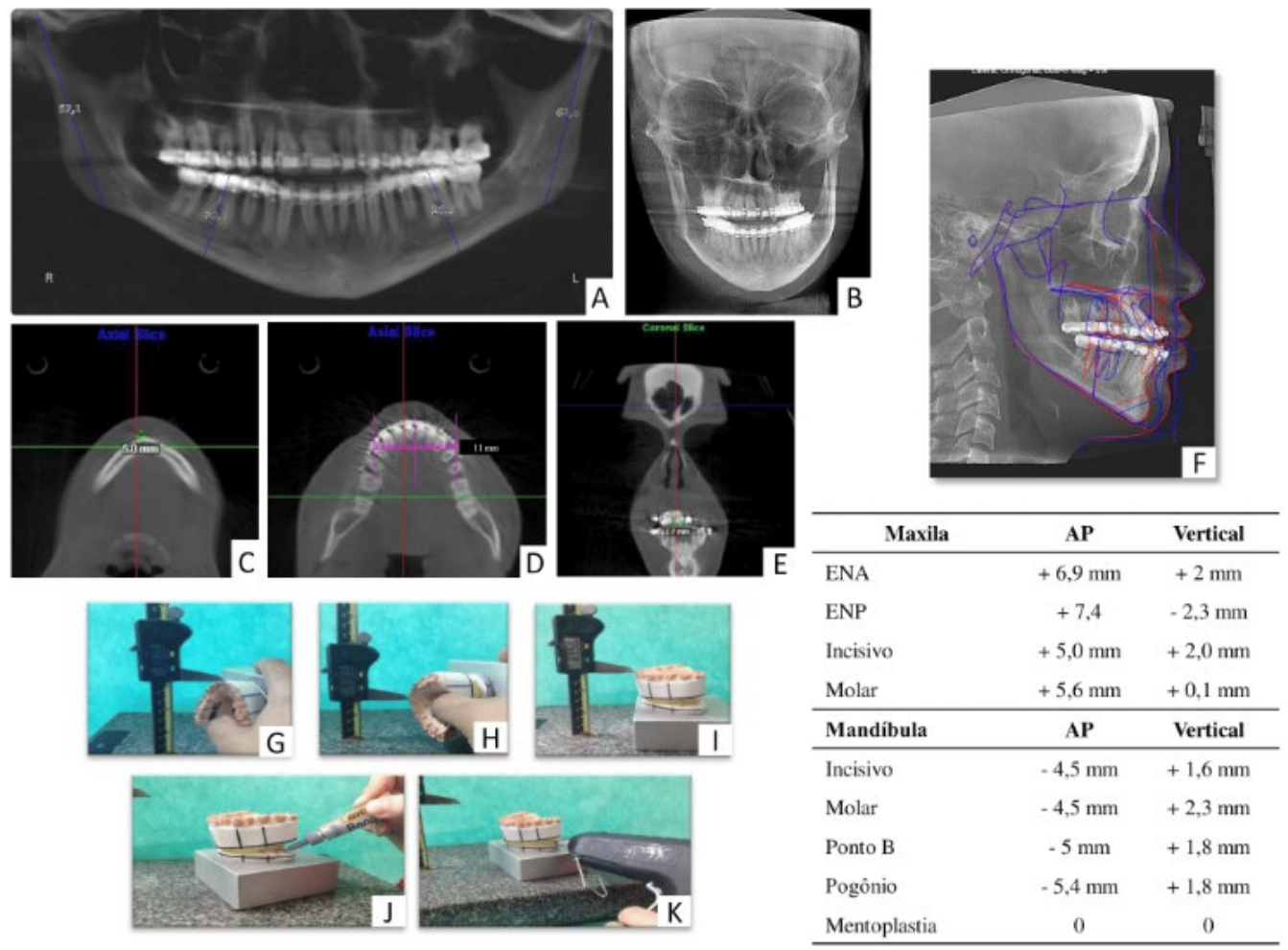

Figura 4: (A-B) Radiografia panorâmica e telerradiografia de perfil apresentaram diferenças de altura entre os ramos mandibulares; (C-D) Reconstruções multiplanares em TCFC para demonstrar desvio em sínfise e linhas médias dentárias; (F) Traçado original e preditivo sobrepostos em telerradiografia de perfil; (G-K) Posicionamento de maxila por meio de plataforma de Erickson para simulação em cirurgia de modelo
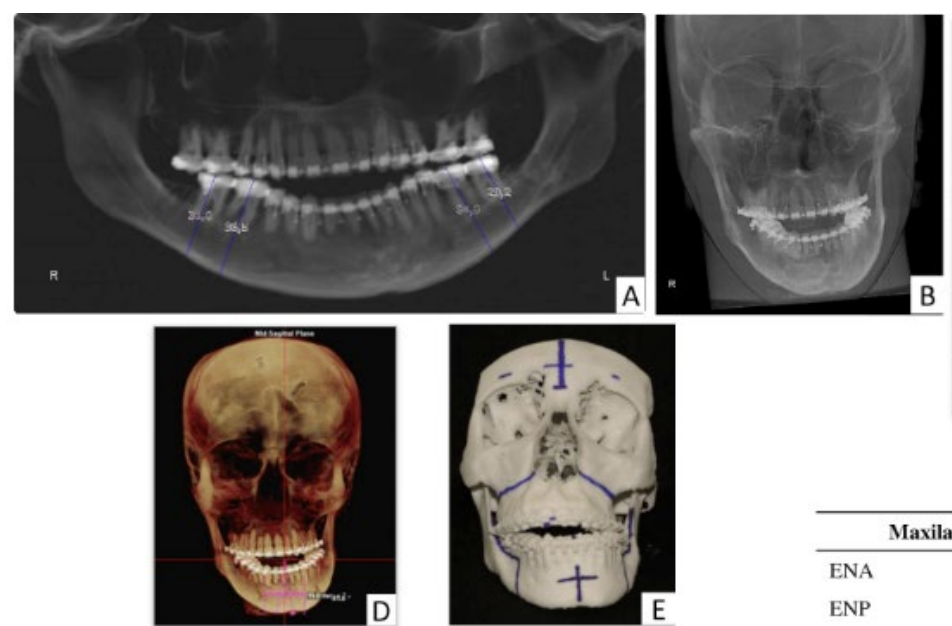

B
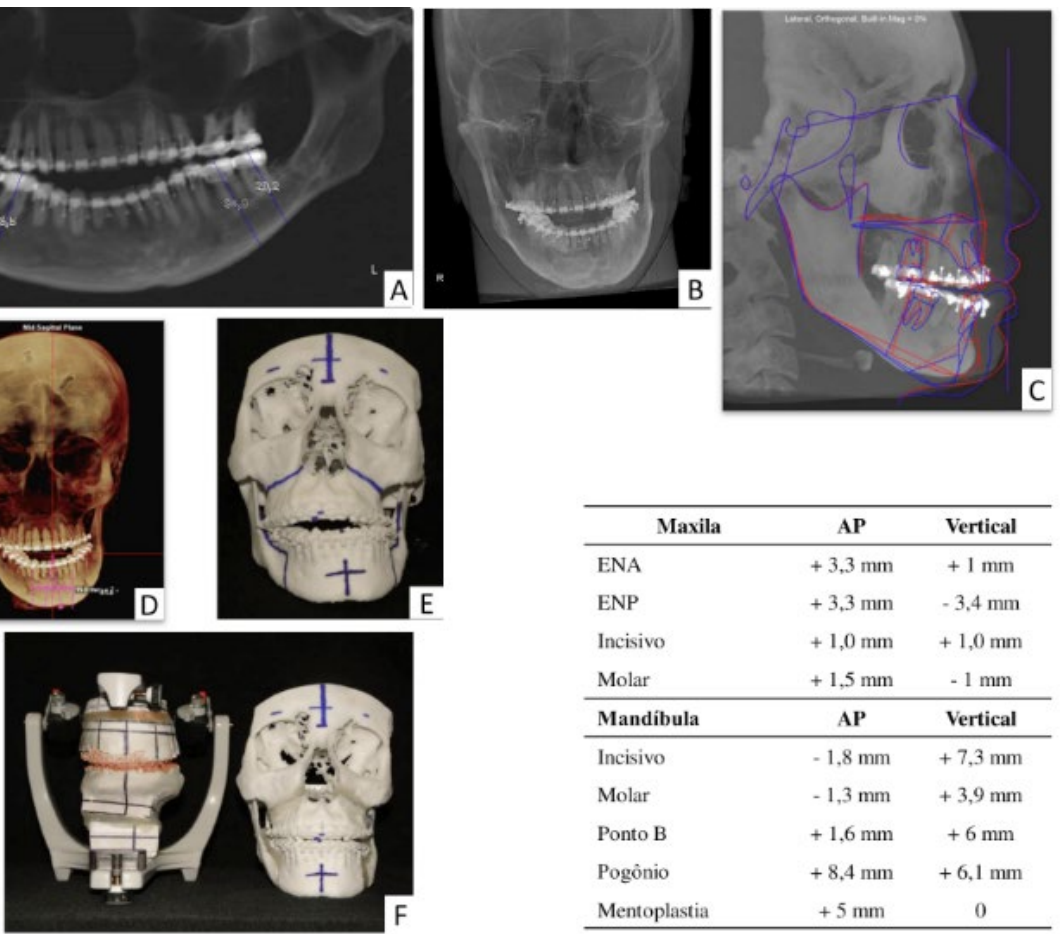

\begin{tabular}{lcc}
\hline \multicolumn{1}{c}{ Maxila } & AP & Vertical \\
\hline ENA & $+3,3 \mathrm{~mm}$ & $+1 \mathrm{~mm}$ \\
ENP & $+3,3 \mathrm{~mm}$ & $-3,4 \mathrm{~mm}$ \\
Incisivo & $+1,0 \mathrm{~mm}$ & $+1,0 \mathrm{~mm}$ \\
Molar & $+1,5 \mathrm{~mm}$ & $-1 \mathrm{~mm}$ \\
\hline Mandibula & $\mathbf{A P}$ & Vertical \\
\hline Incisivo & $-1,8 \mathrm{~mm}$ & $+7,3 \mathrm{~mm}$ \\
Molar & $-1,3 \mathrm{~mm}$ & $+3,9 \mathrm{~mm}$ \\
Ponto B & $+1,6 \mathrm{~mm}$ & $+6 \mathrm{~mm}$ \\
Pogônio & $+8,4 \mathrm{~mm}$ & $+6,1 \mathrm{~mm}$ \\
Mentoplastia & $+5 \mathrm{~mm}$ & 0 \\
\hline
\end{tabular}

Figura 5: (A-B) Imagem tridimensional das estruturas ósseas maxilomandibulares pré-operatórias Radiografia panorâmica e telerradiografia de perfil apresentaram diferenças de altura entre os ramos mandibulares; (C) Traçado original e traçado preditivo sobrepostos em telerradiografia de perfil; (D-E) Reconstrução tridimensional e protótipo confeccionado; (F) Simulação dos movimentos em cirurgia de modelo e protótipo 
O acesso vestibular da mandíbula foi executado através da incisão em tecido mole sobre a linha obliqua externa até a mesial do segundo molar, lateralmente (mínimo de $5 \mathrm{~mm}$ de mucosa não ceratinizada mantida em região de vestíbulo). Foi efetuada, então, dissecção subperiosteal da mucosa bucal em direção à linha obliqua interna na área retromolar com intuito de exposição parcial da região medial e língula da mandíbula. Através de serras reciprocantes (Stryker - CORE System), a osteotomia sagital da mandíbula bilateral foi feita, finalizada por uso de cinzeis. Os guias cirúrgicos em resina acrílica então foram fixados aos acessórios ortodônticos para bloqueio maxilomandibular com fio de aço. Mandíbula e maxila foram estabilizadas em oclusão final, sendo a mandíbula posicionada através de fixação interna rígida com miniplacas retas e parafusos monocorticais (Sistema 2.0 - Neoface - Neoortho Produtos Ortopédicos).
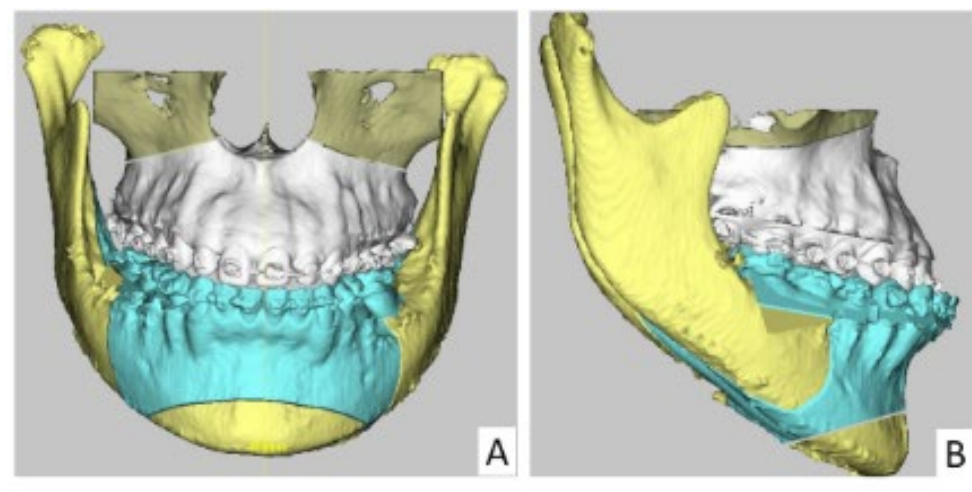

\begin{tabular}{|c|c|c|c|c|}
\hline \multicolumn{4}{|c|}{ Landnark Olfsets Measurements } & \multirow{2}{*}{ Total } \\
\hline Landark. & AP & LA & Veit & \\
\hline - Marda [Model BLock] & P./A+ & $\mathrm{R}-\mathrm{l}$ - & Down + & Tots \\
\hline BNS & +327 & 0.59 & -171 & 374 \\
\hline PNS & +3.23 & -3.57 & +0.22 & 4.82 \\
\hline Upper Incisor Tp Midooin. & +4.58 & +2.02 & -201 & 5.39 \\
\hline U3 Conine Tip (L] & +3.38 & +1.38 & .290 & 4.66 \\
\hline U3 Canine Tip (R) & +5.65 & +1.21 & .0 .31 & 578 \\
\hline U6 Mesial Cusp Tip (l) & +256 & 0.37 & .263 & 369 \\
\hline U6 Mesial Cusp Tip [R] & +6.15 & 0.55 & $\bullet 1.41$ & 6.33 \\
\hline -Mandble [Model BLock] & P./A+ & $\mathrm{A} \cdot \Omega$. & $\mathrm{Up}_{p}+$ & $T \alpha a$ \\
\hline Lover Incisor Tp Midpont & -4.43 & -1.83 & +499 & 692 \\
\hline L6 Metial Cusp Ip (L) & -3.46 & -0.08 & +2.87 & 4.50 \\
\hline L6 Mesial Cusp Tb [R] & .6 .53 & +0.12 & -1.54 & 6.71 \\
\hline Bfoint & .297 & -0.36 & +406 & 504 \\
\hline Pogonion & +2.19 & +1.03 & +415 & 4.81 \\
\hline Gerioplasty & +3.00 & 0 & -0.06 & 300 \\
\hline$-\operatorname{Madla}(\mathrm{A} \mid)$ & P./A. & $R \cdot \Omega$. & Down. & Tod \\
\hline APoint & +3.46 & 0.27 & .171 & 387 \\
\hline
\end{tabular}
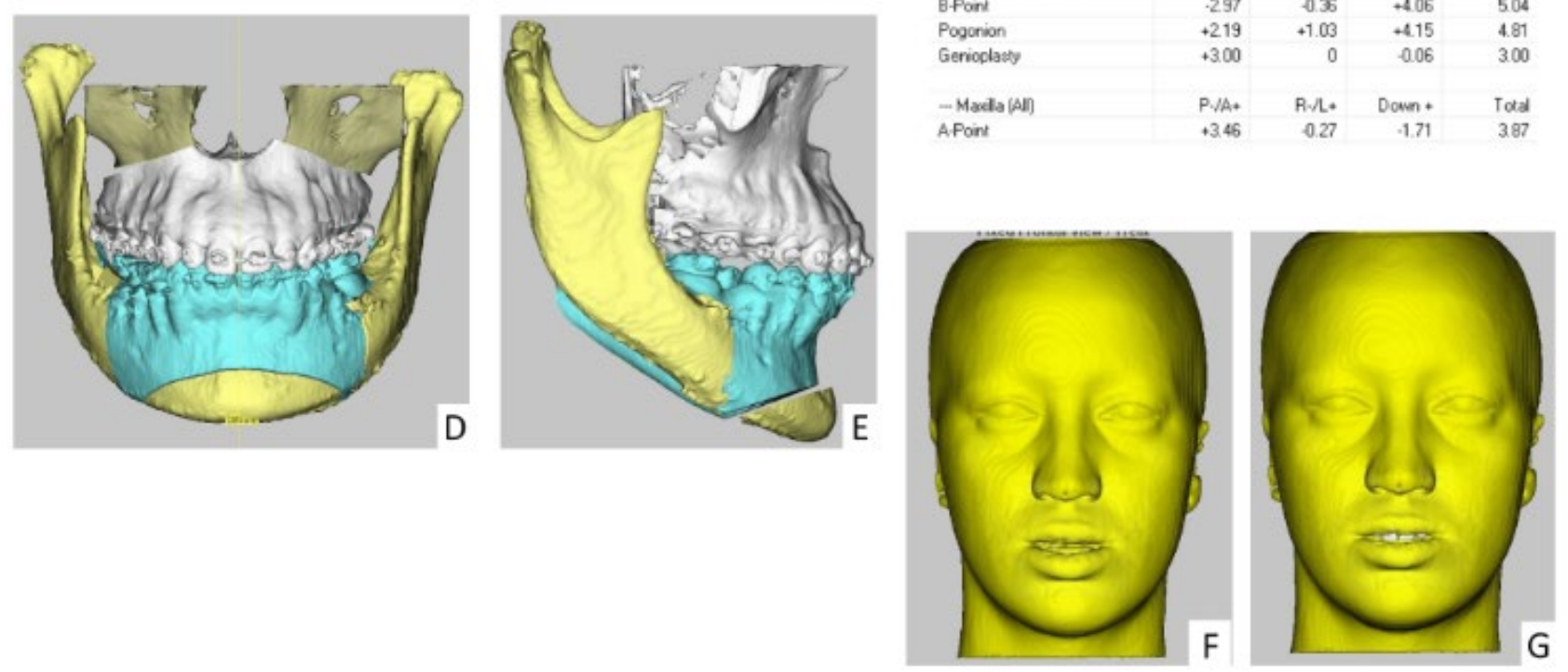

Figura 6: (A-B) Reconstrução 3D de maxila e mandíbula prévio a simulação de planejamento virtual; (C) Valores em mm das movimentações esqueléticas e dentárias em maxila e mandíbula; (D-E) Reconstrução 3D de maxila e mandíbula após simulação de planejamento virtual; (F-G) Contorno de tecido mole prévio e após a simulação

Para os casos B e C, as mesmas condutas mencionadas foram realizadas, mas em sequência invertida, sendo a mandíbula operada primeiramente em relação à maxila. E exclusivamente para o caso $C$, foram utilizados guias estereolitográficos provenientes da simulação cirúrgica virtual. Para esses dois casos, foram realizados mentoplastia para avanço de mento e melhora em contorno de sulco mentolabial.

\section{Pós-operatório}

Os pacientes foram avaliados semanalmente nos primeiros 2 meses e mensalmente até o sexto mês. Os tratamentos ortodônticos pós-operatórios foram mantidos até a finalização.

Foi constatado, por parte dos próprios pacientes, grau elevado de satisfação, com ótimo resultado estético e funcional. Os resultados dos casos A, B e C permaneceram estáveis após um ano de pós-operatório (Figuras 7, 8 e 9).

\section{DISCUSSÃO}

Previamente aos planejamentos e cirurgias, todos os três pacientes foram submetidos ao preparo ortodôntico, o que permitiu um acompanhamento clínico da assimetria facial por período de 06 meses. Foi excluída a realização 
de exames de cintilografia e tomografia por emissão de pósitrons para avaliar as regiões de articulações temporomandibulares, pois acredita-se que os resultados poderiam ser inespecíficos, sendo as evidências clínicas (idade, acompanhamento radiográfico e fotográfico, e modelos de gesso) de inatividade da HC mais determinantes para não progressão da condição. Dessa forma, os casos relatados evidenciaram assimetrias faciais que foram causadas por $\mathrm{HC}$ unilateral caracterizada por uma atividade de crescimento acentuado em côndilo mandibular.

Os parâmetros aplicados nos relatos foram similares ao de Wolford; Movahed; Perez (2014), que relataram que as $\mathrm{HC}$ ocorrem usualmente durante a puberdade, ocasionando prognatismo mandibular (má-oclusão Classe III), mordida cruzada posterior, desvio de linha média dentária inferior e mentoniana, o que altera a morfologia mandibular. Em certas ocasiões, haverá pacientes com efeitos residuais desse crescimento anormal em maxila (desnivelamento oclusal e desvio de linha média). Acrescentam considerarem que a cintilografia ou tomografia por emissão de pósitrons são desnecessárias para avaliar hiperatividade na $\mathrm{HC}$, pois a característica de progressão de assimetria facial até meados dos 20 anos fundamenta-se como uma condição autolimitante, e os cirurgiões devem avaliar clinicamente os pacientes pelo período de 06 a 12 meses antes do
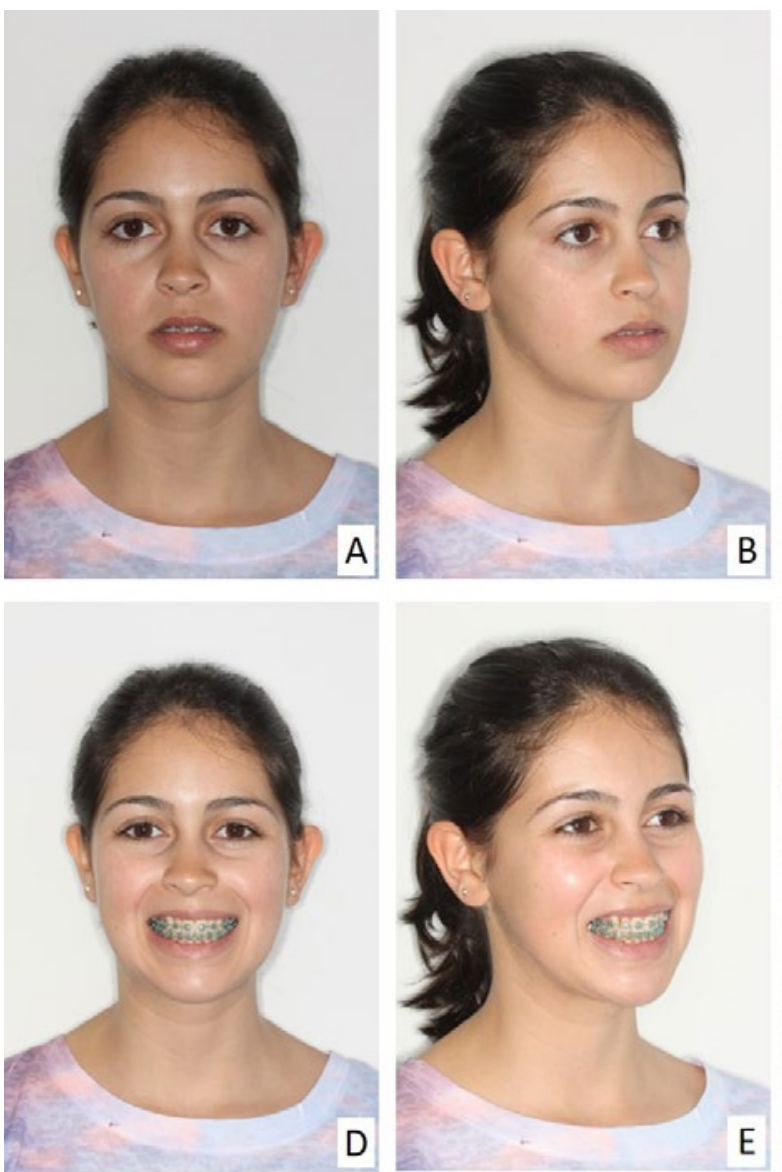

procedimento cirúrgico.

O tratamento de HC unilateral deve ser definido de acordo com a progressão ou não de atividade de crescimento. Os relatos apresentaram inatividade quanto a HC em pacientes com mais de 20 anos de idade, restringindo-se a correção cirúrgica da assimetria facial com intuito de oferecer resultados estéticos e funcionais. Em nenhum dos casos foi realizado condilectomia alta prévia ou concomitante a cirurgia ortognática. De acordo com Wolford; Movahed; Perez (2014), se os pacientes já se encontram na fase de inatividade quanto ao crescimento por $\mathrm{HC}$, a cirurgia ortognática usualmente poderá ser realizada para correção da deformidade dentofacial sem necessidade de abordagem em côndilo mandibular.

Ghawsi; Aagaard; Thygesen (2016) revisaram sistematicamente a eficácia da condilectomia alta para tratamento de $\mathrm{HC}$, destacando que este procedimento é controverso pelo caráter autolimitante da condição de crescimento acentuado, o que indicaria um possível adiamento de abordagem cirúrgica. Entretanto, se os pacientes em fase de crescimento desejarem a correção da deformidade dentofacial por aspectos psicossociais e desajuste social (SUEN et al., 2018), é valido considerar a condilectomia alta para cessar a condição hiperplásica na mandíbula e minimizar recidivas após a cirurgia ortognática (LÓPEZ; CORRAL, 2015).
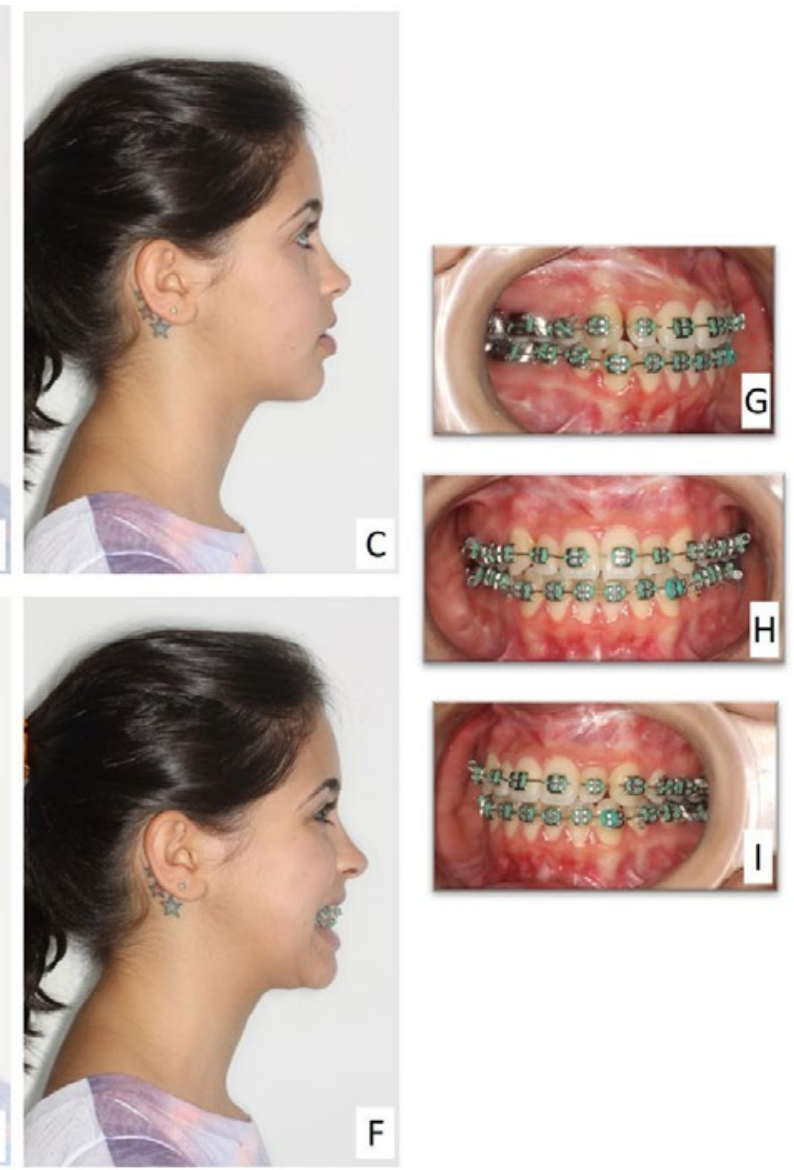

Figura 7: (A-F) Imagens do resultado pós-operatório em repouso e ao sorriso; (G-I) Imagens intraorais da oclusão em pós-operatório 

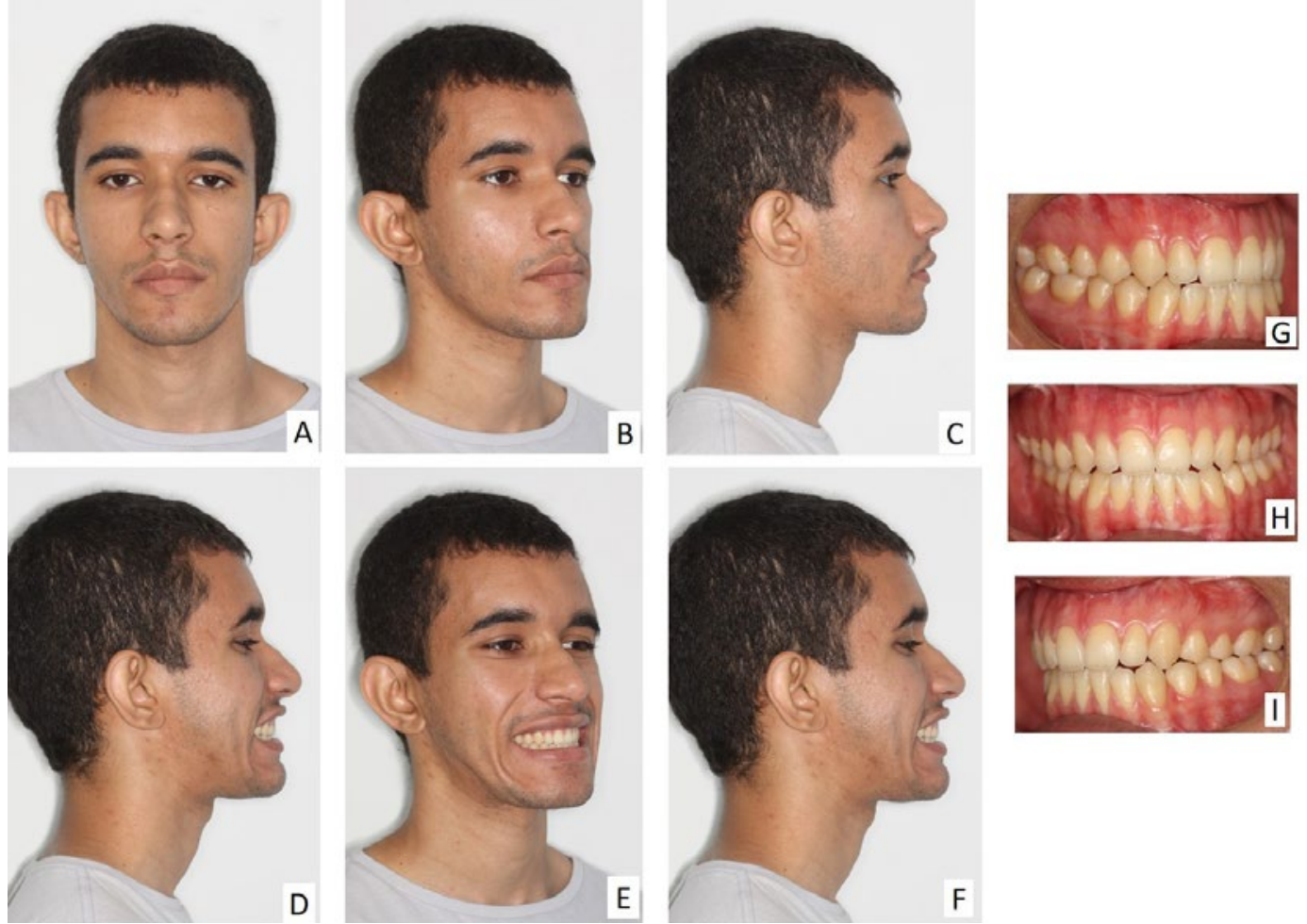

Figura 8: (A-F) Imagens do resultado pós-operatório em repouso e ao sorriso; (G-I) Imagens intraorais da oclusão em pós-operatório
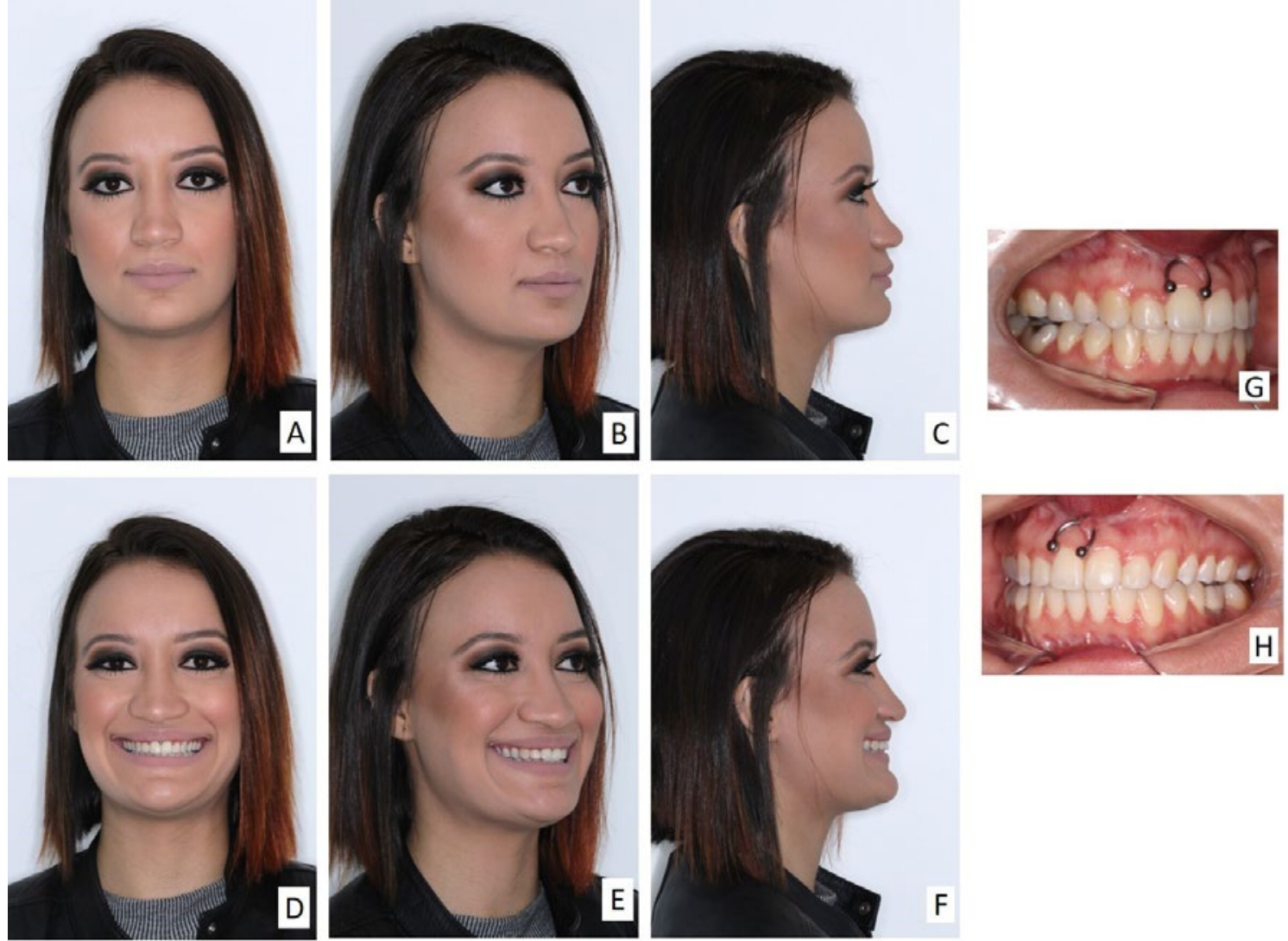

Figura 9: (A-F) Imagens do resultado pós-operatório em repouso e ao sorriso; (G-I) Imagens intraorais da oclusão em pós-operatório 
Para a execução da cirurgia ortognática, é fundamental desenvolver planejamentos prévios aos procedimentos cirúrgicos. Esses planejamentos podem ser caracterizados como convencionais, caracterizado por fluxo de trabalho descrito nos casos A e B, que utilizaram imagens 2D, traçados preditivos e cirurgia de modelo em ASA. Nesse sentido, Ritto et al. (2018) destacaram que os planejamentos convencionais têm sido realizados há mais de 50 anos, sendo o fluxo de trabalho reprodutível e os resultados obtidos em pós-operatórios favoráveis. No entanto, o planejamento convencional pode acumular pequenos erros durante o fluxo de trabalho, desde a aquisição de modelos de gesso e montagem em ASA inadequadas até confecção de guias cirúrgicos insatisfatórios, o que levaria a imprecisões importantes. Assim, o planejamento convencional apresenta possíveis erros cumulativos do processo laboratorial até a visualização 3D das estruturas ósseas, principalmente, quando a deformidade dentofacial é complexa, como assimetria facial (RITTO et al., 2018).

Para minimizar essa limitação do planejamento convencional em assimetrias faciais, o caso B utilizou o recurso de imagem 3D da TCFC para avaliação da disposição das estruturas ósseas, e prototipagem para simular os movimentos cirúrgicos dos segmentos osteotomizados previamente a cirurgia ortognática. Embora, este recurso aumente mais uma etapa laboratorial no planejamento, acredita-se que o protótipo permitiu verificar o comportamento de maxila e mandíbula a partir dos guias confeccionados, que auxiliaram a transferência do planejamento e eram provenientes da simulação da cirurgia de modelo, que se limitou a apresentar as relações entre os arcos maxilo-mandibulares.

O planejamento virtual elimina muitas etapas laboratoriais quando comparado ao fluxo de trabalho do convencional, o que tende a diminuir tempo dispendido prévio a cirurgia, minimiza erros cumulativos e apresenta resultados mais precisos (KWON et al., 2014). O relato C realizou exclusivamente um planejamento virtual, excluindo traçados preditivos em imagens 2D, montagem em ASA de modelos de gesso e cirurgia de modelo. Todas as etapas foram realizadas a partir da renderização 3D da TCFC em programas computacionais, que permitiu simular osteotomias em maxila e mandíbula, bem como, visualizar os movimentos ósseos e a influência sobre os tecidos moles.

Em todos casos aqui relatados, os procedimentos cirúrgicos foram realizados pela mesma equipe de cirurgiões por meio de técnicas padronizadas. A única diferença para o caso $A$ foi a sequência cirúrgica iniciada pela maxila em relação a iniciada pela mandíbula para os casos B e C. Entende-se que a sequência iniciada pela maxila favoreceu ao planejamento de rotação no sentido horário do complexo maxilomandibular, mas poderia dificultar a correção da assimetria, porque a posição final assumida pela mandíbula (maior proporção assimétrica) seria dependente da posição da maxila. Os três casos apresentavam semelhanças clínicas pré-operatórias, contudo, os casos B e C demonstraram melhores resultados qualitativos quanto à simetria mandibular, e parece que a sequência iniciada pela mandíbula facilitou a correção da região com maior proporção assimétrica. Além disso, a sequência iniciada pela mandíbula é benéfica para rotações anti-horárias do complexo maxilomandibular (Casos B e C), mordida aberta anterior, imprecisão do registro intercondilar e incerteza quanto ao posicionamento do côndilo (MARLIÈRE et al., 2018).

Em relação a precisão do planejamento, desde a utilização dos traçados cefalométricos para planejamento em cirurgia ortognática, o desvio máximo de $2 \mathrm{~mm}$ era aceitável entre o plano de tratamento e resultados obtidos para tecidos moles e esqueléticos (MARQUETTI et al., 2006). Atualmente, muitos autores evidenciam que os critérios de sucesso e clínico aceitável ainda permanecem se a diferença máxima de $2 \mathrm{~mm}$ entre o planejado e obtido em pós-operatório (STOKBRO et al., 2014). Apesar de não ter sido feito nenhuma mensuração quanto a precisão dos diferentes planejamentos e resultados, os casos foram qualitativamente satisfatórios. Os casos aqui apresentados sugerem que não houve diferenças na resolutividade funcional e estética, independente do planejamento executado. Essas evidências podem ser confirmadas pelo estudo retrospectivo de Ritto et al. (2018), que compararam o planejamento virtual e convencional por meio de mensurações lineares na maxila em TCFC de 30 pacientes submetidos a cirurgia ortognática, os resultados não demonstraram diferenças entre os planejamentos e resultados pós-operatórios obtidos.

Portanto, concordamos que os avanços tecnológicos quanto ao planejamento em cirurgia ortognática aumentaram a acurácia dos resultados em casos de assimetria, alinhamento de linhas médias e posicionamento mandibular quando comparados ao planejamento convencional (RIU et al. 2014). No entanto, o planejamento virtual não exclui a importância do cirurgião quanto ao plano de tratamento baseado em uma análise facial criteriosa, percepção clínica para otimização funcional e estética, fluxo de trabalho padronizado, e execução cirúrgica tecnicamente consolidada.

\section{CONCLUSÃO}

Os diferentes planejamentos em cirurgia ortognática demonstraram previsibilidade e foram viáveis para aplicação em pacientes assimétricos, proporcionando resultados satisfatórios para os casos relatados. Para os casos $B$ e $C$, houve uma tendência de melhores resultados porque recursos 3D ou imagens 3D foram utilizados nos planejamentos e as cirurgias empregaram a sequência operatória iniciada pela mandíbula. 


\section{REFERÊNCIAS}

GHAWSI, S.; AAGAARD E.; THYGESEN, T. H. High condylectomy for the treatment of mandibular condylar hyperplasia: a systematic review of the literature. International Journal of Maxillofacial Surgery, v. 45, n. 1, p. 60-71, jan. 2016.

GOOD, S. et al. A computerized photographic assessment of the relationship between skeletal discrepancy and mandibular outline asymmetry. European Journal of Orthodontics, v. 28 , n. 2, p. 97-102, jan. 2006

HAAS JR, O. L.; BECKER, O. E.; OLIVEIRA, R. B. Computeraided planning in orthognathic surgery- systematic review. International Journal of Oral \& Maxillofacial Surgery, v. 44, p. 329-342, nov. 2014.

KWON, T. G. et al. Accuracy of maxillary repositioning in two-jaw surgery with conventional articulator model surgery versus virtual model surgery. International Journal of Oral \& Maxillofacial Surgery, v. 43, n. 6, p. 732-738, jan. 2014.

LÓPEZ, D. F. B.; CORRAL, C. M. S. Condylar Hyperplasia: characteristics, manifestations, diagnosis and treatment. A topic review. Revista Faculdad de Odontología Universidad de Antioquia, v. 26, n. 2, p. 425-446, mar. 2014.

MARLIÈRE, D. A. A. et al. Orthognathic surgery combined with partial lipectomy of the buccal fat pad: case report on optimization of esthetic outcome. Journal of Pharmacy and Pharmacology, v. 5, p. 565-571. 2017.

MARLIÈRE, D. A. A. et al. Alteration of occlusal plane in orthognathic surgery: clinical features to help treatment planning on class III patients. Case Reports in Dentistry, v. 2018, p. 1-9, may. 2018.

MARQUETTI, C. et al. Mathematical modeling and numerical simulation in maxilla-facial virtual surgery (VISU). Journal of Craniofacial Surgery, v. 17, n. 4, p. 661-667, jul. 2006.

RAMIREZ-YAÑEZ, G. O. et al. Prevalence of mandibular asymmetries in growing patients. European Journal of Orthodontics, v. 33, n. 3, p. 236-242, jun. 2011.

RITTO, F. G. et al. Comparison of the accuracy of maxillary position between conventional model surgery and virtual surgical planning. International Journal of Oral \& Maxillofacial Surgery, v. 47, n. 2, p. 160-166, feb. 2018.

RIU, G. D. et al. Computer-assisted orthognathic surgery for correction of facial asymmetry: results of a randomised controlled clinical trial. British Journal of Oral and Maxillofacial Surgery, v. 52, n. 3, p. 251-257, jan. 2014.
SINGH, V. et al. Orthognathic surgery: a Review of Articles published in 2014-2015. Journal of Maxillofacial and Oral

Surgery, v. 16, n. 3, p. 284-291, sep. 2017.

SRIVASTAVA, D. et al. Facial asymmetry revisited: part Idiagnosis and treatment planning. Journal of Oral Biology and Craniofacial Research, v. 8, n. 1, p. 7-14, apr. 2018.

SRIVASTAVA, D. et al. Facial asymmetry revisited: Part IIConceptualizing the management. Journal of Oral Biology and Craniofacial Research, v. 8, n. 1, p. 15-19, apr. 2018.

STOKBRO, K. et al. Virtual planning in orthognathic surgery. International Journal of Oral and Maxillofacial Surgery, v. 43 , n. 8 , p. 957-965, aug. 2014

SUEN, K. S. et al. A longitudinal evaluation of psychosocial changes throughout orthognathic surgery. PLoS One, v. 13, n. 9, p. $1-12$, sep. 2018

THIESEN, G.; GRIBEL, B. F.; FREITAS, M. P. M. Facial asymmetry: a current review. Dental Press Journal of Orthodontics, v. 20, n. 6 , p. 110-125, dec. 2015

SUN, Y. et al. Accuracy of upper jaw positioning with intermediate splint fabrication after virtual planning in bimaxillary orthognathic surgery. The Journal of Craniofacial Surgery, v. 24, n. 6, p. 1871-1876, nov. 2013.

WOLFORD, L. M. et al. Surgical management of mandibular condylar hyperplasia type 1. Archive of Proceedings (Baylor University Medical Center), v. 22, n. 4, p. 321-329, oct. 2009.

WOLFORD, L. M.; MOVAHED, R.; PEREZ, D. E. A classification system for conditions causing condylar hyperplasia. Journal of Oral and Maxillofacial Surgery, v. 72, n. 3, p. 567-595, mar. 2014. 